GANIT J. Bangladesh Math. Soc. (ISSN 1606-3694) 32 (2012) 15 - 21

\title{
A NEW APPROACH TO SOLVE FUZZY NON-LINEAR EQUATIONS USING FIXED POINT ITERATION ALGORITHM
}

\author{
Goutam Kumar Saha and Shapla Shirin ${ }^{1}$ \\ Department of Mathematics, University of Dhaka \\ Email: goutam.math@gmail.com and shapla@univdhaka.edu
}

Received 21-10-2011 Accepted 03-11-2012

\begin{abstract}
A non-linear equation over linear fuzzy real numbers is called a fuzzy non-linear equation. In Classical Mathematics a non-linear equation can be solved by using different types of numerical methods. In this paper a new approach has been introduced to get approximate solutions with the help of Fixed Point Iteration Algorithm. Graphical representation of the solutions has also been drawn so that anyone can achieve the idea of converging to the root of a fuzzy non-linear equation.
\end{abstract}

Keywords: Fixed Point Iteration, Fuzzy non-linear equation, Linear Fuzzy Real Number, $n$-th root of a fuzzy number.

\section{Introduction}

In algebra, it is easy to solve literal equations $f(x)=0$ of all degrees up to and including fourth. But we are not always able to get exact solution of equations. Besides this, no general method exists for finding the roots of this equation in terms of their co-efficient. It is always possible to compute the roots to any desired degree of accuracy. A method has been discussed to solve a fuzzy linear equation directly [4]. But this method is not capable of computing of a solution of FNLE. In this paper a new idea has been introduced to solve a fuzzy non-linear equation with the help of Fixed Point Iteration Algorithm. An example is also discussed and the approximate solutions, which are LFRNs, computed from each iteration are shown in a tabular form. The graphical representations of these approximate solutions of the fuzzy non-linear equation are depicted to achieve the idea of converging to the root of the FNLE. Finally, the precise solution of the fuzzy non-linear equation is obtained with the help of the proposed algorithm and its graphical representation has also been shown as well.

Some related definitions and some operations on linear fuzzy real numbers [3, 5-10], which will be used later to obtain a solution of fuzzy nonlinear equation to any desired degree of accuracy, has been given in the following section.

\section{Preliminaries}

In this section some definitions have been discussed which are important to us for representing our main objective in the later sections. 
Definition 2.1 [8] (Linear fuzzy real number, LFRN): Let $\mathcal{R}$ be the set of all real numbers and $\mu: \mathcal{R} \rightarrow[0,1]$ be a function such that $[8]$

$$
\mu(x)=\left\{\begin{array}{l}
0 \text { if } x \leq a \text { or } x \geq c \\
\frac{x-a}{b-a} \text { if } a<x<b \\
1 \quad \text { if } x=b \\
\frac{c-x}{c-b} \text { if } b<x<c
\end{array}\right.
$$

Then $\mu(a, b, c)$ is called a linear fuzzy real number with associated triple of real numbers $(a, b, c)$ where $a \leq b \leq c$ shown in Fig.1.1.

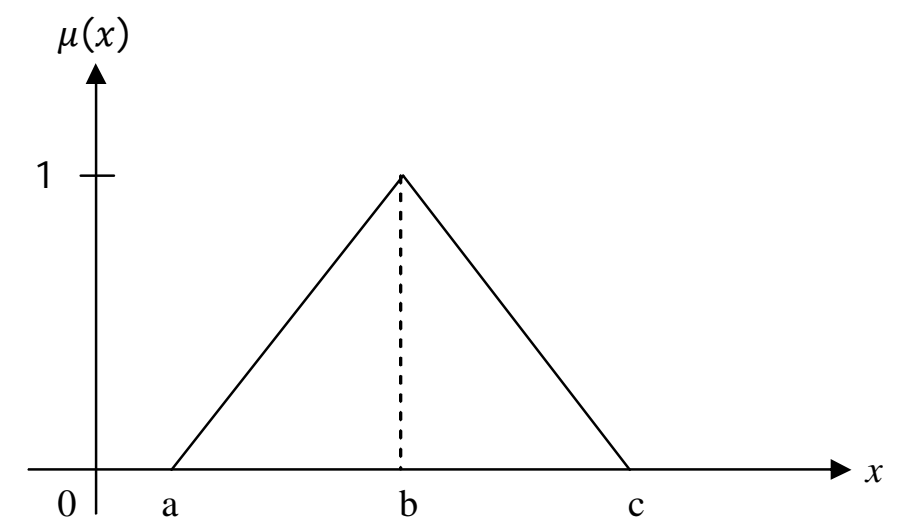

Fig 1.1 Linear fuzzy real number $\mu(a, b, c)$.

Let $L F^{\mathcal{R}}$ be the set of all linear fuzzy real numbers. We note that any real number b can be written as a linear fuzzy real number, $(b)$, where $r(b)=\mu(b, b, b)$ and therefore $\mathcal{R} \subseteq L F^{\mathcal{R}}$. Now it is clear that $r(b)$ represents the real number b itself. Operations on LFRN, fuzzy functions and fuzzy linear equations in $L F^{\mathcal{R}}$ are also defined in $L F^{\mathcal{R}}$ as follows.

Definition 2.2 [8] (Operations on $L F^{\mathcal{R}}$ ) : For given two linear fuzzy real numbers $\mu_{1}=\mu\left(a_{1}, b_{1}, c_{1}\right)$ and $\mu_{2}=\mu\left(a_{2}, b_{2}, c_{2}\right)$, we define addition, subtraction, multiplication and division by

1. $\mu_{1}+\mu_{2}=\mu\left(a_{1}+a_{2}, b_{1}+b_{2}, c_{1}+c_{2}\right)$;

2. $\mu_{1}-\mu_{2}=\mu\left(a_{1}-c_{2}, b_{1}-b_{2}, c_{1}-a_{2}\right)$;

3. $\mu_{1} \cdot \mu_{2}=\mu\left(\min \left\{a_{1} a_{2}, a_{1} c_{2}, a_{2} c_{1}, c_{1} c_{2}\right\}, b_{1} b_{2}, \max \left\{a_{1} a_{2}, a_{1} c_{2}, a_{2} c_{1}, c_{1} c_{2}\right\}\right)$;

4. $\frac{\mu_{1}}{\mu_{2}}=\mu_{1} \cdot \frac{1}{\mu_{2}}$, where $\frac{1}{\mu_{2}}=$

$\mu\left(\min \left\{\frac{1}{a_{2}}, \frac{1}{b_{2}}, \frac{1}{c_{2}}\right\}\right.$, median $\left.\left\{\frac{1}{a_{2}}, \frac{1}{b_{2}}, \frac{1}{c_{2}}\right\}, \max \left\{\frac{1}{a_{2}}, \frac{1}{b_{2}}, \frac{1}{c_{2}}\right\}\right)$.

Definition 2.3 [8] (Function in $L F^{\mathcal{R}}$ ) : If $f: \mathcal{R} \rightarrow \mathcal{R}$ is a real-valued function and $\mu(a, b, c)$ is a LFRN, then the linear fuzzy real-valued function $\bar{f}: L F^{\mathcal{R}} \rightarrow L F^{\mathcal{R}}$ is defined as

$$
\bar{f}(\mu(a, b, c))=\mu(\bar{a}, \bar{b}, \bar{c}),
$$


Where $\bar{a}=\min \{f(a), f(b), f(c)\}, \bar{b}=\operatorname{median}\{f(a), f(b), f(c)\}, \bar{c}=$ $\max \{f(a), f(b), f(c)\}$. We note that if $a=b=c$ then $\bar{a}=\bar{b}=\bar{c}$, i.e., $\bar{f}(r(b))=$ $r(f(b))$. Hence $\bar{f}$ is an extension of the function $f$.

Definition 2.4 [8] (Linear equation in $L F^{\mathcal{R}}$ ) : A linear equation over $L F^{\mathcal{R}}$ is an equation of the form

$$
\mu_{1} \cdot \mu_{x}+\mu_{2}=\mu_{3},
$$

where the $\mu_{i}$ are $L F R N$ s for $i=1,2,3$ with a triple of unknown real numbers $\left(\alpha_{i}, \beta_{i}, \gamma_{i}\right)$.

Definition 2.5 [1] (Square root of a fuzzy number) : Square root of a fuzzy number $\mu(a, b, c)$ is defined as

$$
\sqrt{\mu(a, b, c)}=\mu(\sqrt{a}, \sqrt{b}, \sqrt{c})
$$

where $a, b, c \geq 0$.

Definition 2.6 [1] (n-th root of a fuzzy number) : n-th root of a fuzzy number $\mu(a, b, c)$ is defined by

$$
\sqrt[n]{(\mu(a, b, c))}=\mu(\sqrt[n]{a}, \sqrt[n]{b}, \sqrt[n]{c})
$$

where $a, b, c \geq 0$.

Definition 2.7 [8] (Fuzzy non-linear equations in $L F^{\mathcal{R}}$ ) : Fuzzy non-linear equations can be found in many applications, all the way from light diffraction to planetary orbits for example. In this section, we discuss how to solve fuzzy non-linear equations in $L F^{\mathcal{R}}$. Thereby a solution $\mu_{x}$ in $L F^{\mathcal{R}}$ of a fuzzy a non-linear equation $\bar{f}\left(\mu_{x}\right)=0$ will be obtained where $\bar{f}: L F^{\mathcal{R}} \rightarrow L F^{\mathcal{R}}$ is a non-linear function.

For example $\mu_{x}^{3}+\mu_{x}^{2}-1=0$ is fuzzy non-linear equation.

\section{The fixed Point Iteration Method to solve Fuzzy Non-linear equation}

Solving fuzzy non-linear equations over $L F^{\mathcal{R}}$ of the form $\bar{f}\left(\mu_{x}\right)=0$ is possible with a modification of Fixed Point Iteration method over linear fuzzy real numbers. This method begins with an initial approximation $\mu_{x}^{(0)}=\mu\left(a^{(0)}, b^{(0)}, c^{(0)}\right) \in L F^{\mathcal{R}}$ and generates the sequence $\left\{\mu_{x}^{(n)}\right\}_{n=0}^{\infty}$ with $\mu_{x}^{(n)}=\mu\left(a^{(n)}, b^{(n)}, c^{(n)}\right)$ by $\mu_{x}^{(n)}=\varphi\left(\mu_{x}^{(n-1)}\right)$, where $\bar{f}\left(\mu_{x}\right)=\mu_{x}-\varphi\left(\mu_{x}\right)=0$ with the condition $\left|\varphi^{\prime}\left(\mu_{x}\right)\right|<1$.

The stopping criterion of this method is $\left|r\left(b^{(n)}-b^{(n-1)}\right)\right|<\epsilon$, where $\epsilon$ is a preset small value (e.g., $\epsilon=0.00001$ ).

\section{Modification of Fixed Point Iteration Algorithm}

Now the question arises, why the modification of existing algorithm is required. This is because of the formation of FNLEs which consist of fuzzy functions and LFRNs, whereas a non-linear equation consists of functions and real numbers.

To find a solution of FNLE $\bar{f}\left(\mu_{x}\right)=\mu_{x}-\varphi\left(\mu_{x}\right)=0$ i.e., $\mu_{x}=\varphi\left(\mu_{x}\right)$, an initial approximation $\mu_{x}^{(0)}=\mu\left(a^{(0)}, b^{(0)}, c^{(0)}\right)$ is assumed. 
INPUT: Initial approximation $\mu_{x}^{(0)}=\mu\left(a^{(0)}, b^{(0)}, c^{(0)}\right) \quad$; tolerance TOL; maximum number of iterations $n$.

OUTPUT: optimum solution $\mu_{x}^{(n)}=\mu\left(a^{(n)}, b^{(n)}, c^{(n)}\right)$ or message of failure.

Step-1: Set $i=1$.

Step-2: While $i \leq n$ do steps $3-6$.

Step-3: Set $\mu_{x}=\varphi\left(\mu_{x}\right) . \quad$ (Compute $\mu_{x}^{(n)}$ )

Step-4: If $\left|\mu\left(b^{n}\right)-\mu\left(b^{n-1}\right)\right|<$ TOL then

$\operatorname{OUTPUT}\left(\mu_{x}^{(n)}\right) . \quad$ (The procedure is successful.)

STOP.

Step-5: Set $i=i+1$.

Step-6: $\mu_{x}^{(n-1)}=\mu_{x}^{(n)} \quad\left(\right.$ Update $\left.\mu_{x}^{(0)}\right)$

Step-7: OUTPUT. ("The method failed after $n$ iteration, $n$ " $n$ );

(The procedure is unsuccessful.)

STOP.

Example. Solve a fuzzy non-linear equation $\mu_{x}^{3}+\mu_{x}^{2}-1=0$.

Solution: Let, $\bar{f}\left(\mu_{x}\right)=\mu_{x}^{3}+\mu_{x}^{2}-1$, then write $\bar{f}\left(\mu_{x}\right)$ in the form $\mu_{x}=\varphi\left(\mu_{x}\right)$, where $\left|\varphi^{\prime}\left(\mu_{x}\right)\right|<1$.If we set $\mu_{x}=\frac{1}{\sqrt{\mu_{x}+1}}$ use an initial approximation $\mu_{x}^{(0)}=\mu(0.5,0.75,1)$ then we get,

$$
\begin{gathered}
\left|\varphi^{\prime}\left(\mu_{x}\right)\right|=\left|-\frac{1}{2\left(\mu_{x}+1\right)^{3 / 2}}\right|=\left|\frac{1}{2 \mu^{3 / 2(1.5,1.75,2)}}\right|=\left|\frac{1}{2 \mu(1.83712,2.31503,2.82843)}\right| \\
=\left|\mu\left(\frac{1}{5.65685}, \frac{1}{4.63006}, \frac{1}{3.67424}\right)\right| \\
=\mu(0.176777,0.21598,0.272165)<1
\end{gathered}
$$

Iteration- $1: n=1$

$$
\begin{aligned}
\mu_{x}^{(1)} & =\frac{1}{\sqrt{\mu_{x}^{(0)}+1}}=\frac{1}{\sqrt{\mu(1.5,1.75,2)}}=\frac{1}{\mu(1.22474,1.32288,1.41421)} \\
& =\mu(0.707107,0.755926,0.8165)
\end{aligned}
$$

Which is an approximation to the solution of FNLE.

Proceeding in this way we can get the solutions shown in the following Table: 
Table: Approximate solutions of fuzzy non-linear equation using Fixed Point Iteration algorithm.

\begin{tabular}{|c|c|c|}
\hline $\begin{array}{c}\text { Iteratio } \\
\text { n no. } \\
\mathrm{n}\end{array}$ & $\mu_{x}^{(n)}$ & $\mu_{x}^{(n)}=\frac{1}{\sqrt{\mu_{x}^{(n-1)}+1}}$ \\
\hline 1. & $\mu_{x}^{(1)}$ & $\mu(0.707107,0.755926,0.8165)$ \\
\hline 2. & $\mu_{x}^{(2)}$ & $\mu(0.741961,0.754654,0.765369)$ \\
\hline 3. & $\mu_{x}^{(3)}$ & $\mu(0.752632,0.754928,0.757673)$ \\
\hline 4. & $\mu_{x}^{(4)}$ & $\mu(0.754279,0.754865,0.755361)$ \\
\hline 5. & $\mu_{x}^{(5)}$ & $\mu(0.754774,0.754882,0.757008)$ \\
\hline 6. & $\mu_{x}^{(6)}$ & $\mu(0.754848,0.754877,0.754899)$ \\
\hline 7. & $\mu_{x}^{(7)}$ & $\mu(0.754871,0.754877,0.754882)$ \\
\hline
\end{tabular}

From iteration 7 we have obtained the solution which is correct up to five decimal places.

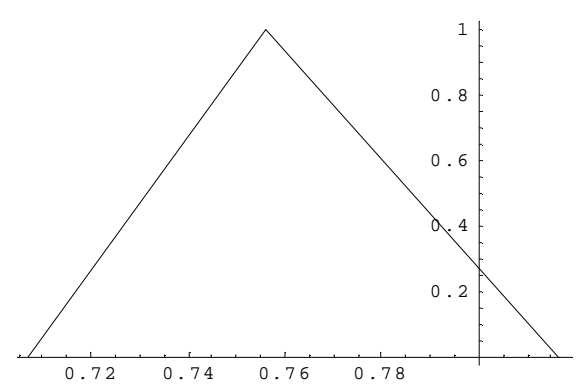

Fig. : Iteration-1

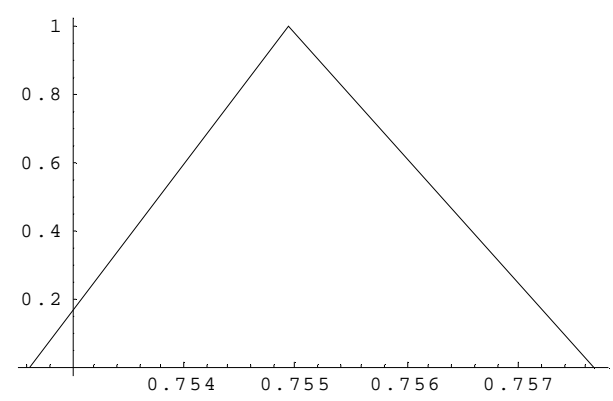

Fig. : Iteration-3

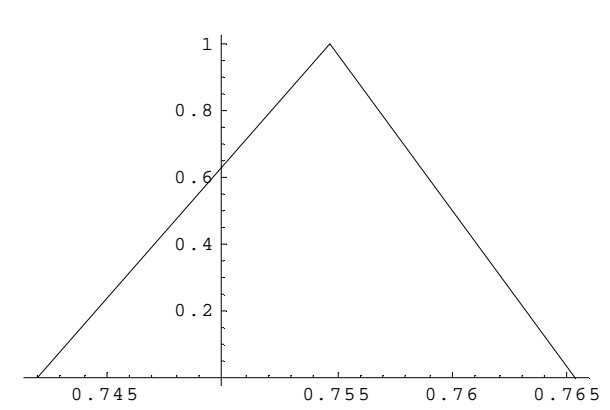

Fig. : Iteration-2

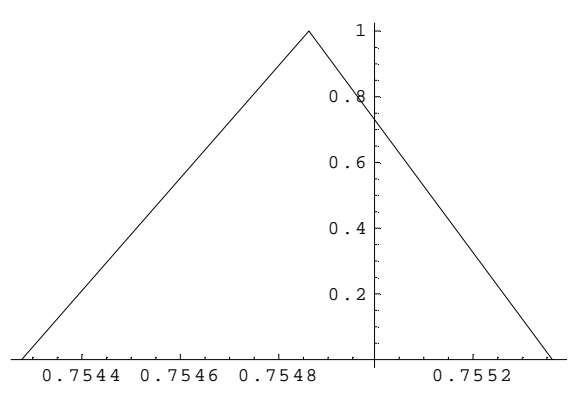

Fig. : Iteration-4 


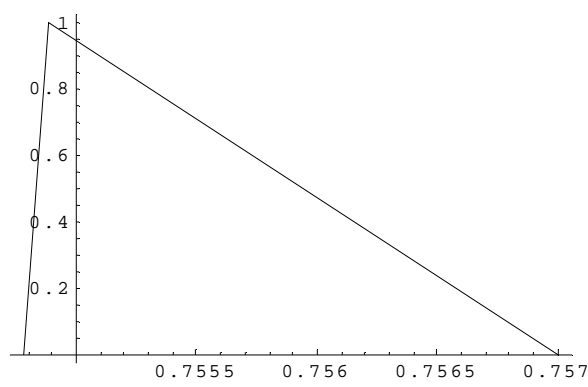

Fig. : Iteration-5

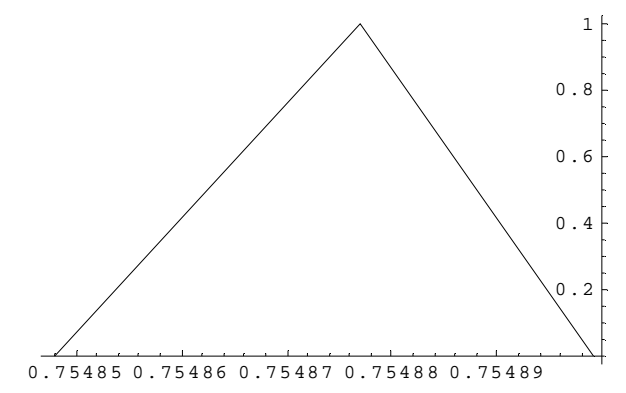

Fig. : Iteration-6

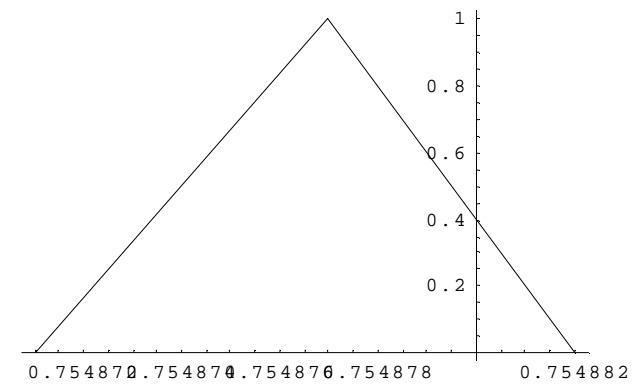

Fig. : Iteration-7

Fig 1.2: Graphical representations of approximate solutions of the given fuzzy non-linear equation.

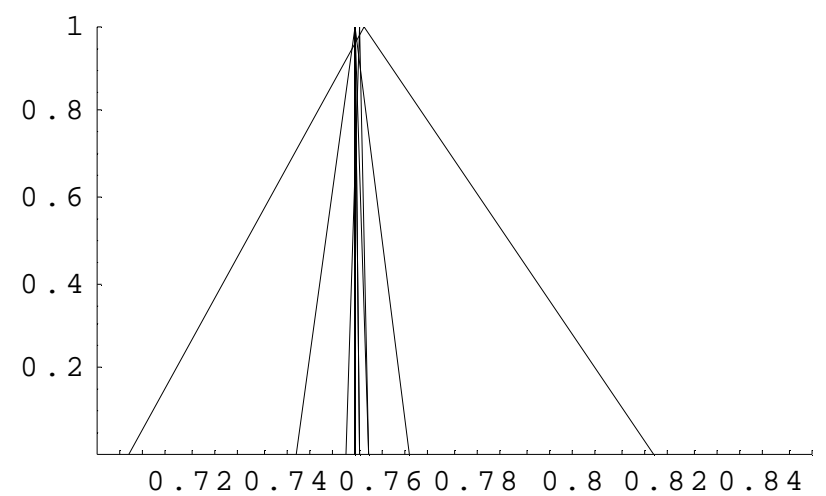

Fig 1.3: Graphical representation of optimum solution of a fuzzy non-linear equation given in the above example. 


\section{Conclusion}

In this paper a new approach has been introduced to solve a fuzzy non-linear equation (FNLE) with the help of Fixed Point Iteration Algorithm. However, by using this new approach a fuzzy non-linear equation (FNLE) is solved and also obtained the solutions of the FNLE to the desired degree of accuracy which is the optimum solution of the given equation. We have also represented the approximate solutions of the FNLE graphically by which one can understand how the solutions converge to the required accuracy.

\section{REFERENCES}

[1] Bashar M.A. and Shirin S., Squares and square roots of continuous fuzzy numbers, Dhaka Univ. J. Sci., 53(2), July 2005,P. 131 - 140.

[2] Burden R. L. and Faires J. D., Numerical Analysis, $7^{\text {th }}$ edition, P-55-63.

[3] Dubois D., Prade H., System of linear fuzzy constraints, Fuzzy Sets and System 13 (1982), P. 1 - 10.

[4] Klir G.J., Yuan B., Fuzzy sets and fuzzy logic Theory and Applications, Prentice-Hall of India Private Limited, New Delhi, (1997), P.1-117.

[5] Neggers J., Kim H., On Linear Fuzzy Real Nmbers, Manuscript for book under development, 2007.

[6] Negoita C.V., Fuzziness in management, OPSA/TIMS, Miami, 1970.

[7] Prevo R, Entropies of families of fuzzy random variables: an introduction to an in-depth exploration of several classes of important examples, Dissertation, University of Alabama, 2002.

[8] Rogers F. and Jun Y., Fuzzy Nonlinear Optimization for the Linear Fuzzy Real Number System, International Mathematical Forum, 4, 2009, no. 12, P. 589 - 596.

[9] Rogers F., Neggers J., Jun Y., Method for optimizing linear problems with fuzzy constrains, International Mathematical Forum 3 (2008), P. 1141 - 1155.

[10] Zimmermann H.J., Fuzzy mathematical programming, Comput. Ops. Res.10 (1983), P. 291 $-298$. 